\title{
The time required to compare extents in various orientations
}

\author{
FRED ATTNEAVE and GENE BLOCK \\ University of Oregon, Eugene, Oregon 97403
}

\begin{abstract}
In Experiment I, Ss compared the lengths of lines that varied in absolute and relative orientation. Their reaction times showed a constant error pattern of which the familiar horizontal-vertical illusion was a special case, but horizontals were underestimated (relative to other slopes) much more than verticals were overestimated. The effect of difference in orientation was not great, though RT did increase significantly as the difference neared $90 \mathrm{deg}$. Experiment II was similar except that the lengths of lines were compared with the widths of bars in a grating. In this case, no consistent pattern of constant error emerged, and there was no suggestion that difference in orientation affected RT.
\end{abstract}

One of the most fundamental capabilities of the visual system is that of evaluating and comparing spatial extents. Objects are located partly in terms of their distances from other objects; moreover, within-object extents, relative to one another, form an important component of shape: e.g., the shape of a rectangle is purely a matter of its length-width ratio. The problem of extent perception is similar to the problem of object identification in this respect: that both are instances of perceptual response invariance over retinal transformations corresponding to the possible translations and rotations of a line or object in physical space. Extent constancy may indeed contribute to object constancy over these transformations.

Most of the work on spatial constancies has been concerned with those cases in which retinal extent is imperfectly correlated with physical extent, because of variations in viewing distance or angle of regard. But a logically prior, and presumably simpler, but nevertheless unanswered question is how the nervous system measures or compares extents that are retinally equivalent, e.g., projections of lines that vary in place and orientation in the frontal plane.

The time required to compare extents in different orientations should give us some information about the mechanism involved. A universally known operation for comparing, say, the length of two sticks is to place them side by side, aligned at one end, and observe which extends beyond the other at the opposite end. It is quite conceivable that the nervous system might operate in an analogous fashion, vicariously rotating one line into the other. This hypothesis gains some minimal plausibility from the convincing evidence reported by Shepard and his associates (Shepard \& Metzler, 1971; Cooper \& Shepard, 1973) that "mental rotation" does occur (in depth as well as in the frontal plane) in the comparison of complex objects. If extent comparisons likewise involve mental rotation, there should be a linear relationship, as in the studies just cited, between the time required for comparison and the amount of rotation necessary to bring one extent into the same orientation as the other.

Another possibility, suggested by the work of Posner and his associates on "identity-matches" vs "name-matches" (Posner \& Mitchell, 1967; etc.), is that extents in the same orientation might be compared at a lower "node" in the perceptual system than extents in different orientations, and require less time for that reason. If this were the case, the time function would very likely be nonlinear, incrasing most rapidly at small angular differences. Further possibilities will be considered in the Discussion.

The task in Experiment I was merely to choose which of two lines in the frontal plane was the longer, over trials varying the absolute and relative slopes of the lines. Experiment II was similar, except that the length of a line was compared with the width of bars in a grating. Reaction times were studied, primarily as a function of the angular difference between the extents being compared, and incidentally as a function of the absolute orientations of the extents.

\section{EXPERIMENT I}

\section{Method}

Subjects. Three University of Oregon female undergraduates served as Ss. They were paid for their participation.

Apparatus and Materials. The stimuli were presented in a tachistoscope, monocularly, at a distance of $81 \mathrm{~cm}$ from $S$ 's eye. The exposure tield was a $22.5-\mathrm{cm}$ square, divided down the middle into equal halves, into which separate stimulus cards could be inserted. The preexposure field was a blank white $22.5-\mathrm{cm}$ square; it, like the exposure field, was illuminated by two $6-\mathrm{W}$ fluorescent tubes concealed at the sides.

On any trial, the line segments to be compared were presented one on each side of the tield. separated by about $23.7 \mathrm{~cm}$. On one side, the line appeared $3.6 \mathrm{~cm}$ above the midpoint of the half-field. on the other side, $3.6 \mathrm{~cm}$ below it; thus the imaginary line joining 
the two stimuli had a slope of $\pm 18 \mathrm{deg}$. (We thought it possible that singular results might occur when the stimulus lines were either equal or perpendicular in slope to the imaginary line connecting the $m$, and wished to separate any such singularities from the special case of the horizontal; however, nothing in the results ever suggested that an interaction of this type made any difference.)

The stimulus lines were cut from black adhesive plastic tape, $.8 \mathrm{~mm}$ wide, with the aid of a magnifying comparator scaled in tifths of a millimeter. Results will be reported for comparisons of only two line lengths, differing by approximately $12 \%$, the longer of which was $21.4 \mathrm{~mm}$ and the shorter, $19 \mathrm{~mm}$. On one-third of the trials, however, the longer of these lines was paired with one still longer, or the shorter with one still shorter by an equivalent proportion, in order to require the $S$ actually to compare the lines presented, and not come to rely on absolute judgments. The $12 \%$ difference was great enough to be discriminated with near-perfect accuracy, regardless of orientation, but small enough to demand close attention.

Lines of each length were individually mounted on white cards in 10 different orientations, including the horizontal, the vertical, and all intermediate 18-deg steps.

Procedure. In the course of the experiment, every slope was paired with every other slope eight times (per $S$ ) and with itself four times. The eight comparisons of a line of slope $i\left(S_{j}\right)$ with a line of slope $j\left(S_{j}\right)$ exhausted the possible combinations of three binary procedural variables: (a) $S_{i}$ longer than $S_{j}$ vs $S_{j}$ longer than $S_{j}$; (b) longer line on left vs longer line on right; and (c) longer line in upper position vs longer line in lower position. For comparisons of $S_{\mathfrak{i}}$ with $S_{\mathfrak{i}}$, Variable a disappeared; hence, counterbalancing was accomplished in four trials.

These comparisons were distributed over four sessions of 100 trials each (or 150, counting the discarded trials involving the longest and shortest lines; see above). Each $S_{j} S_{j}$ pair occurred twice per session, with $S_{j}$ longer on one trial and $S_{j}$ longer on the other. and each $S_{i} S_{i}$ pair once per session. The ordering of pairs was random within the first session; this random order was used again on Session 3 and reversed on Sessions 2 and 4. The four states of Procedural Variables $b$ and $c$ were in effect randomly assigned to sessions.

An individual trial proceeded as follows. After changing stimuli, E told S, "Ready." $S$ then pressed a button under his left hand which simultaneously extnguished the preexposure field, illuminated the stimuli, and started a clock. As quickly as possible thereafter, $\mathrm{S}$ moved a key switch with his right hand in the direction (right or left) of the line that he judged to be the longer. This response stopped the clock and restored the preexposure field.

\section{Results}

Before looking at reaction time (RT) as a function of difference in orientation, let us consider how the RTs might reflect constant errors (CEs) of judged length associated with particular absolute orientations. The horizontal-vertical illusion, i.e., the tendency of verticals to look longer than horizontals, will serve as a familiar example to illustrate such a $\mathrm{CE}$ effect. If the $S$ is comparing a long vertical with a short horizontal, the illusion will add to the real difference and make a correct judgment easier and presumably faster. In comparing a long horizontal with a short vertical, however, the illusion will subtract from the real difference and make the judgment more difficult and presumably slower. The disparity between these two RTs should therefore provide a measure of the horizontal-vertical illusion. More generally, the greater the CE difference favoring $S_{i}$ over $S_{j}$, the greater will be the difference, RT $\left(S_{i}<S_{j}\right)$ minus RT $\left(S_{i}>S_{j}\right)$, always assuming that the illusion does not exceed the real difference.
Finally, the mean of the differences, RT $\left(S_{\mathfrak{i}}<S_{\mathfrak{j}}\right)$ minus RT $\left(S_{i}>S_{j}\right)$, over all values of $S_{j}$, may be taken as an ordinal measure of the $C E$ associated with $S_{i}$, with positive values indicating that $S_{i}$ is overestimated in extent relative to other orientations, and negative values indicating that it is underestimated.

This mean difference, which we shall call simply the "CE index," was calculated for all 10' line orientations, for individual Ss. The three Ss show remarkably similar CE patterns; see Fig. 1. There is indeed a horizontal-vertical illusion, but it is not symmetrical about $45 \mathrm{deg}$ : the indifference point is close to $36 \mathrm{deg}$, for all Ss, and the horizontal is underestimated more than the vertical is overestimated. For two of the three Ss, overestimation is greatest 18-36 deg away from the vertical.

RTs for every possible difference in orientation between paired lines $(0,18 \ldots 90 \mathrm{deg})$ were averaged over the various absolute orientations of the components. These means, for individual Ss, are plotted in Fig. 2. The curves are approximately flat over the first half or more, but all three show a modest upturn as the angle approaches $90 \mathrm{deg}$. This increase can be shown to be significant by any one of several tests, perhaps the fairest of which is a comparison of the mean $\log \mathrm{RT}$ of the first three angular differences $(0,18,36)$ with that of the subsequent three $(54,72$, 90) using a between-S error term. The comparison gives a $\mathrm{t}(2)=20.6$, which is significant at the .001 level. Linearity was tested by comparing the mean log RT of the four high and low angular differences $(0$, $18,72,90)$ with that of the two intermediate ones $(36$, 54), this being the ends-vs-middle grouping (consistent with symmetry requirements) that most nearly equates number of observations. The result was a $\mathrm{t}(2)=6.3, \mathrm{p}<.02$. which provides some confirmation for the impression that the curves are concave upward.

It is clear enough that the increase in $\mathrm{RT}$ with angular difference is not attributable to random variability, but we have had some difficulty convincing ourselves that it could not be a complicated artifact of constant error. Illusory effects were ostensibly balanced out by averaging every trial on which $S_{i}$ was longer than $S_{j}$ with an exactly similar trial on which $S_{j}$ was longer than $S_{i}$. However, if difficulty of judgment is dependent on difference in jnds, a subtraction of the CE difference from the real one will always increase difficulty more than an addition of the two will decrease it: this becomes perfectly obvious in the hypothetical case of a $\mathrm{CE}$ difference as great as the real difference. The nonlinearity of RT as a measure of difficulty is not the basic problem, but further compounds it. Note also that the problem is not one of finding an error term that is appropriate, because the disturbing factor is not a neglected source of random variability but a nonrandom bias. This bias would still be a matter of 

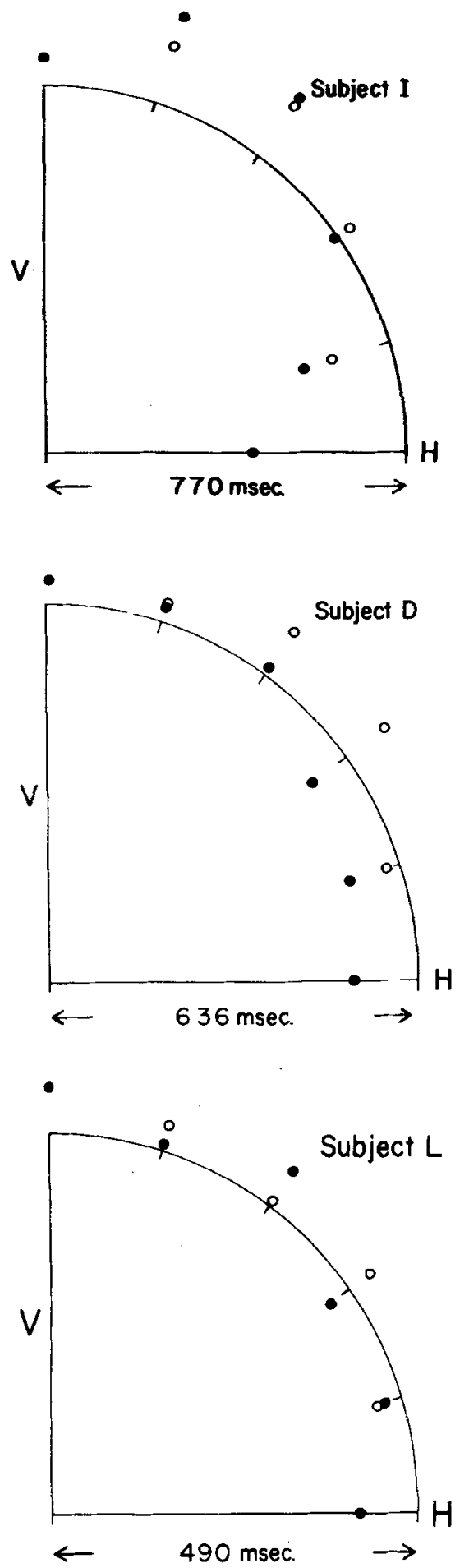

Fig. 1. Constant error index (see text) as a function of absolute line orientation; Experiment $I$. The angular location of a point directly indicates the orientation of the represented line in the case of filled circles; open circles refer to the corresponding negative slopes. CE indices are plotted as deviations from the reference arc (negative inside, positive outside) on a normalized scale that equates the radius of the reference arc to the $S$ 's overall mean reaction time. It should be understood that distances of points from the center of the are are by no means proportional to the apparent lengths of the lines represented; in this sense, the graphs greatly exaggerate constant error.

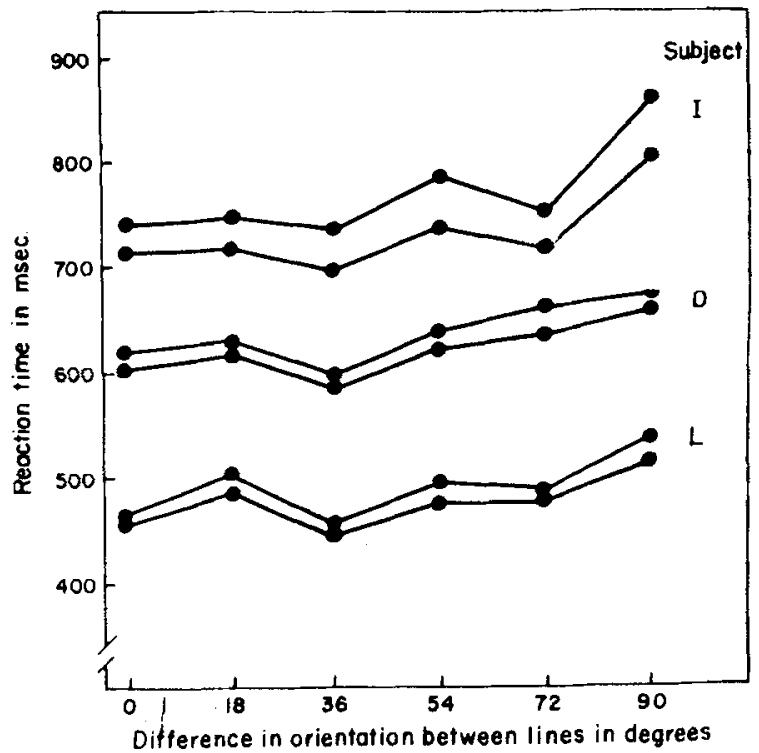

Fig. 2. Comparison time as a function of angular difference between lines, averaged over all absolute orientations; Experiment I. For each S, the upper curve shows arithmetic means, the lower geometric means. Each data point is based on 80 comparisons except for those at 0 and $90 \mathrm{deg}$, which are based on 40 comparisons.

indifference if it were equal for all angular separations, but it is not: Fig. 1 confirms what is virtually inevitable a priori, that, on the average, the CEdifference will be greater for larger angular separations than for smaller ones. The bias accordingly creates a somewhat artificial tendencythe magnitude of which is not easy to evaluate-for $\mathrm{RT}$ to increase with difference in orientation.

Suppose, however, that we deliberately avoid counterbalancing, and consider only those trials (half the total) on which the CE difference, as determined from the data of Fig. 1, was in the same direction as the physical difference, and therefore shortened RT. Since CE differences increase, on the average, with angular separation, RTs should accordingly decrease, other things being equal. In fact, the curves from these unbalanced data (plotted as in Fig. 2) do descend gently from 0 deg to the 36-72-deg region, but then reverse and go up again at the end. A test of the final increase in mean $\log$ RT from 72 to $90 \mathrm{deg}$ yields a $t(2)=4.45, p<.05$, despite the CE bias working in the counter direction. Thus it appears very likely that the increase is genuine; what remains puzzling is its locus.

Error data were carefully examined, but being sparse and unstable they yielded little or no information. Over the 400 trials, S I. made 23 errors, S D., 12, and S L., 21. (Substitute trials were run for all these.) S I.'s errors showed some tendency to increase with angular difference, but this was not the case with either S D. or S L. There was only a weak tendency for errors to occur where they would be 
ATTNEAVE AND BLOCK

expected from illusory effects, e.g., in comparing a long horizontal with a short vertical.

\section{EXPERIMENT II}

In this experiment, $\mathrm{S}$ was required to judge spatial extent in a sense more abstract than formerly, by comparing the length of a line with the width of bars in a grating. We cannot be entirely sure that $S$ was judging extent at all in Experiment I, since the long line was a $12 \%$ greater dark area than the short one, and likewise entailed a $12 \%$ greater amount of contour or dark-light interface. Obviously, neither of these variables would require mental rotation. The aim of Experiment II was to eliminate such perfectly correlated variables and insure that extent per se was the basis of comparison.

\section{Method}

Subjects. Three new undergraduates, one male and two female, were employed and paid for their participation.

Apparatus and Materials. The apparatus was the same as in Experiment 1. Materials consisted of a set of lines and an exactly corresponding set of bar gratings, varying in orientation in steps of $18 \mathrm{deg}$, as before. The extents (line length and bar width) were smaller than in Experiment $I$ to allow a reasonable number of bars to appear in a $9.5-\mathrm{cm}$ circle, but still differed by $12 \%$. On the trials that yielded data, the extents were 6.6 and $7.4 \mathrm{~mm}$, though, as before, these were interspersed with trials in which the greater $(7.4 \mathrm{~mm})$ extent was paired with one still greater $(8.3 \mathrm{~mm})$, or the smaller $(6.6 \mathrm{~mm})$ with one still smaller $(5.9 \mathrm{~mm})$ by the same proportion. The line and the circular grating were centered in their respective halves (left or right) of the exposure field.

Procedure. This experiment introduced a variable (bars vs lines) not present in Experiment 1 , but eliminated a variable (vertical displacement) that was formerly present, so that counterbalancing was accomplished in the same number of trials, i.e., 400 comparisons per $S$ distributed over four sessions. For every possible pairing of orientations, there were eight trials per $S$, the combinations of three binary variables: (a) $S_{i}>S_{j}$ vs $S_{j}>S_{i}$, (b) line greater vs bars greater, and (c) left greater vs right greater; except for comparisons at the same orientation, of which four exhausted the possibilities.

The conduct of individual trials was exactly as before.

\section{Results}

In view of the possibility that constant error as a function of slope might be quite different for line lengths and bar widths, we calculated $C E$ indices separately for lines and bars. CE index was defined earlier as the mean of the differences, $R T\left(S_{i}<S_{j}\right)$ minus RT $\left(S_{i}>S_{j}\right)$, over all values of $j$ : this is now descriptive of either line lengths or bar widths, whichever $i$ is associated with.
To our considerable surprise, neither lines nor bars showed any significant or consistent variation in CE with orientation. The CE indices are listed in Tables 1 and 2 ; they do not graph into any interpretable pattern. The mean indices for lines are all negative, and those for bars are nearly all positive, indicating a strong tendency for lines to be underestimated relative to bars generally. However, there is at best the barest suggestion of consistent illusory effects in Table 1 , and none at all in Table 2. For orientation effects, $F(9,570)=1.39$, n.s., in the case of lines, and $F(9,570)=1.16$, n.s., in the case of bars. Note also the lack of agreement between values at mirror-image slopes: to take an extreme example, the lowest mean index in Table 1 is at $162 \mathrm{deg}$, whereas the mirror-image line of $18 \mathrm{deg}$ has the highest mean but one.

Neither do the results (Fig. 3) show any evidence of increasing RTs with increasing difference in orientation between line and bars. The curves of two Ss are almost completely flat; any trend that one can imagine in their results is in the nonexpected direction. In the case of the third $S$, who was slower and also had a much higher error rate than the other two, the mean RTs are more variable, but show no interpretable trend.

We again examined distribution of errors for possible effects of illusion or of angular difference, with negative results. Errors were somewhat more frequent than in Experiment I: over the 400 trials, Ss B., D., and M. made 35, 19, and 24 errors, respectively.

\section{DISCUSSION}

It is fairly clear that the results do not support either of the hypotheses that we set out to test. If comparison of extents depended on mental rotation, we should find an approximately linear increase in RT with difference in orientation, and nothing of the sort is evident. If comparison were possible at either of two "nodes," the lower of which required near equivalence of orientation and the higher of which did not, RTs should increase most rapidly at small differences in orientation, whereas the only increase that did occur was near the opposite end of the function.

The reason for this increase-why it is found only in line-to-line comparisons (Fig. 2; cf. Fig. 3) and, more

Table 1

CE Indices for Lines of Various Slopes: Experiment II

\begin{tabular}{|c|c|c|c|c|c|c|c|c|c|c|}
\hline \multirow[b]{2}{*}{$\mathrm{S}$} & \multicolumn{10}{|c|}{ Slope (Deg) } \\
\hline & Hor. & 18 & 36 & 54 & 72 & Vert. & 108 & 126 & 144 & 162 \\
\hline $\begin{array}{l}\mathrm{B} \\
\mathrm{D} \\
\mathrm{M}\end{array}$ & $\begin{array}{r}-90 \\
-170 \\
-39\end{array}$ & $\begin{array}{r}32 \\
-41 \\
-18\end{array}$ & $\begin{array}{r}-10 \\
-60 \\
14\end{array}$ & $\begin{array}{r}-60 \\
5 \\
41\end{array}$ & $\begin{array}{r}-36 \\
-6 \\
-19\end{array}$ & $\begin{array}{r}49 \\
-94 \\
-84\end{array}$ & $\begin{array}{r}34 \\
-172 \\
-68\end{array}$ & $\begin{array}{l}-45 \\
-75 \\
-19\end{array}$ & $\begin{array}{r}-75 \\
-172 \\
-54\end{array}$ & $\begin{array}{r}-74 \\
-167 \\
-72\end{array}$ \\
\hline Mean & -100 & -9 & -19 & -5 & -20 & -43 & -69 & -46 & -100 & -104 \\
\hline
\end{tabular}


Table 2

CE Indices for Bars of Various Slopes: Experiment II

\begin{tabular}{|c|c|c|c|c|c|c|c|c|c|c|}
\hline \multirow[b]{2}{*}{$S$} & \multicolumn{10}{|c|}{ Slope (Deg) } \\
\hline & Hor. & 18 & 36 & 54 & 72 & Vert. & 108 & 126 & 144 & 162 \\
\hline $\begin{array}{l}\text { B } \\
\text { D } \\
M\end{array}$ & $\begin{array}{r}-73 \\
130 \\
87\end{array}$ & $\begin{array}{l}65 \\
88 \\
53\end{array}$ & $\begin{array}{l}82 \\
58 \\
52\end{array}$ & $\begin{array}{r}12 \\
182 \\
22\end{array}$ & $\begin{array}{r}-144 \\
57 \\
32\end{array}$ & $\begin{array}{r}60 \\
4 \\
33\end{array}$ & $\begin{array}{r}95 \\
37 \\
-21\end{array}$ & $\begin{array}{r}67 \\
153 \\
15\end{array}$ & $\begin{array}{r}-50 \\
52 \\
70\end{array}$ & $\begin{array}{r}172 \\
197 \\
-19\end{array}$ \\
\hline Mean & 48 & 69 & 64 & 72 & -18 & 32 & 37 & 78 & 24 & 117 \\
\hline
\end{tabular}

Note-."Slope" refers to slope of the extent being compared, which is at right angles to the bars.

particularly, why it appears only when the lines approach a right angle with each other - is totally obscure to us, and follows from no plausible hypothesis that we have entertained. We can only confess our bafflement in this matter, and hope that the reader may have an insight that has eluded us.

However, the fact that difference in orientation has so little effect on the speed of discriminating extents would seem to indicate that the visual system is capable of measuring in many directions at once and projecting these measurements to a common continuum. (Cf. the Structuralist view of extensity as an attribute of sensation.) In terms of formal mechanisms, this might mean either that the measuring device is inherently indifferent to orientation, like a series of circular apertures graded in diameter, or that orientation-specific measuring devices are grouped by extent (and, presumably, grouped separately by slope) at a higher level. The latter possibility is compatible with various theories which hold that the visual system performs something like a spatial Fourier analysis on its input (Kabrisky, Tallman, Day, \& Radoy, 1970; Pollen, Lee, \& Taylor, 1971).

Other evidence argues strongly for the parallel processing of extent information. Harrington (1967) found that very brief exposure was sufficient for a short line to be located in a field of long ones, even when the latter varied among themselves in both slope and thickness, and that the task became no more difficult, but perhaps easier, as the number of lines in the field was increased (see also Olson \& Attneave, 1970). Likewise, results of Attneave and Frost (1969, also Attneave, 1972), which indicate that objects tend to be seen in depth in such a way as to equalize perceived extents (the lengths of edges), would be very hard to reconcile with a theory that extents are evaluated serially, e.g., by pairwise comparisons requiring rotation to a common orientation.

Perhaps the most intriguing difference in results of the two present experiments is with respect to constant error: the highly consistent CE pattern found in Experiment I (Fig. 1) did not appear in Experiment 11. The results of the latter do not, of course, prove that the null hypothesis is true, and Table 1 does show some suggestion of an effect. These results are nevertheless so unimpressive, and in such sharp contrast to those of Experiment I, as to suggest that the illusory effects in the first study are not to be attributed to any general anisotropy of visual space, but rather to some more specific factor, presumably associated with comparisons between lines. This might be some variable that provides a basis for comparing lines with lines, but not lines with bars, which could further account for the generally shorter RTs in Experiment I than in Experiment II. One plausible candidate for this role, suggested earlier, is amount of contour, which was perfectly correlated with extent in Experiment I but not in Experiment II. The level of activity in a nerve net characterized by lateral inhibition, or receptive fields with centersurround antagonism, will vary with amount of contour, and might therefore be used as a measure of length (not necessarily to the exclusion of all other criteria) in the special case of comparisons between lines. But if the receptive fields deviated systematically from rotational symmetry, the same line would appear longer, by this measure, in some orientations than in others.

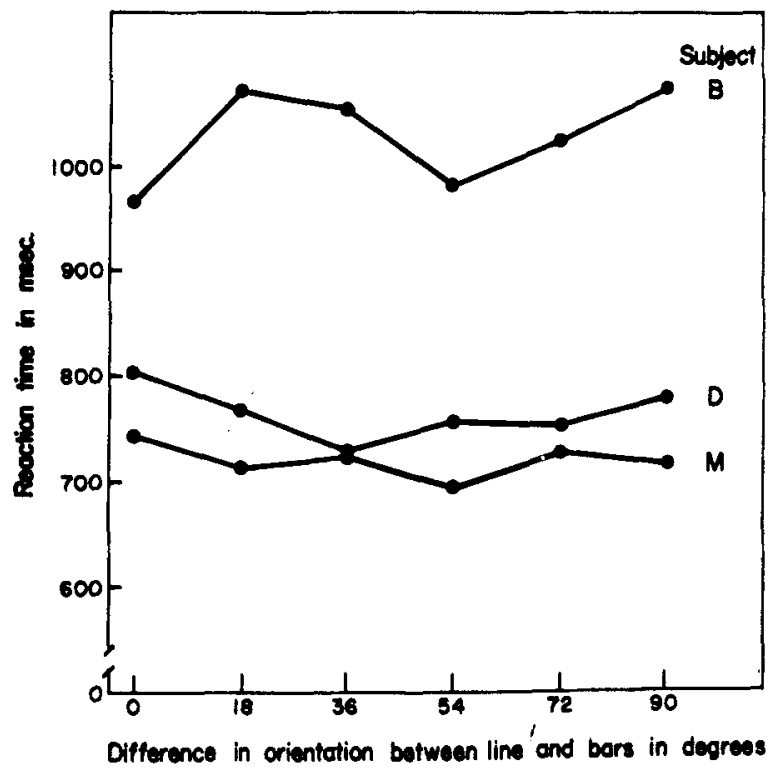

Fig. 3. Comparison time as a function of angular difference between line and bars; arithmetic means; Experiment II. At zero difference, the extents being compared were in the same orientation; thus the line was not parallel but perpendicular to the bars. Data base as in Fig. 2. 


\section{REFERENCES}

Attneave, F. The representation of physical space. In A. W. Melton and E. J. Martin (Eds.). Coding processes in human memory. Washington, D.C: Winston, 1972.

Attneave, F., \& Frost, R. The determination of perceived tridimensional orientation by minimum criteria. Perception \& Psychophysics, 1969, 6, 391-396.

Cooper, L. A., \& Shepard, R. N. Chronometric studies of the rotation of mental images. In W. G. Chase (Ed.), Visual information processing. New York: Academic Press, 1973.

HaRrington, T. L. Analysis of line length by the human visual system. Doctoral dissertation, University of Oregon, 1967.

Kabrisky, M., Tallman, O., Day, C. M., \& Radoy, C. M. A theory of pattern perception based on human physiology. Ergonomics, 1970, 13, 129-147.
Olson, R. K., \& Atrneave, F. What variables produce similarity grouping? American Journal of Psychology, 1970, 83, 1.21 .

Pollen, D. A., lee, J. R., \& Taylor, J. H. How does the striate cortex begin the reconstruction of the visual world? Science, 1971, 173, 74-77.

Posner, M. I., \& Mrtchell, R. F. Chronometric analysis of classification. Psychological Review, 1967, 74, 392-401.

Shepard, R. N., \& Metzler, J. Mental rotation of threedimensional objects. Science, 1971, 171, 701-703.

(Received for publication March 8, 1974; revision received June 12,1974 .) 